\title{
Factores predictores del estado libre de cálculos en la primera ureteroscopia flexible con litotricia láser
}

\author{
Efraín Maldonado-Alcaraz, ${ }^{1}$ Mario Alberto Ramírez-Negrín, ${ }^{1}$ Juan Rodríguez-Silverio, ${ }^{2}$ \\ León Octavio Torres-Mercado, ${ }^{1}$ Virgilio Augusto López-Sámano, ${ }^{1}$ Guillermo Montoya-Martínez ${ }^{1}$ y \\ Jorge Moreno-Palacios ${ }^{1}$ \\ IInstituto Mexicano del Seguro Social, Centro Médico Nacional Siglo XXI, Hospital de Especialidades; ${ }^{2}$ Instituto Politécnico Nacional, Escuela \\ Superior de Medicina, Sección de Estudios de Posgrado e Investigación. Ciudad de México, México
}

\section{Resumen}

Introducción: La ureteroscopia flexible con litotricia láser (URSLL) es una modalidad mínimamente invasiva de tratamiento quirúrgico de cálculos renales. La selección inadecuada de pacientes para este procedimiento genera un desbalance de costo-efectividad. Objetivo: Conocer los factores predictores de estado libre de litos en un solo tiempo quirúrgico en pacientes sometidos a URSLL. Método: Cohorte retrospectiva de pacientes sometidos a URSLL. Se realizó un análisis univariado y multivariado (regresión logística) de los predictores de estado libre de cálculos en la primera URSLL, global y categorizado por sexo. Resultados: El estado libre de cálculos en la primera URSLL fue de $73.62 \%$. Los predictores de estado libre de cálculos en hombres fueron edad y tamaño, densidad y multiplicidad del cálculo; en las mujeres, el índice de masa corporal y la multiplicidad del cálculo. Conclusiones: Los factores pronósticos de estado libre de cálculos en la primera URSLL son distintos en hombres y mujeres. Las mujeres con obesidad y sobrepeso probablemente tengan cálculos de fácil fragmentación y extracción asociados con ácido úrico.

PALABRAS CLAVE: Ureteroscopia. Cálculos renales. Resultado del tratamiento. Costos y análisis de costos. Obesidad.

\begin{abstract}
Introduction: Flexible ureteroscopy and laser fragmentation (FURSL) is a minimally invasive modality for surgical treatment of renal stones. Inadequate selection of patients for this treatment generates a cost-effectiveness unbalance. Objective: To know the stone-free rate predictors in a single surgical time in patients undergoing FURSL. Method: Retrospective cohort of patients undergoing FURSL. Global and gender-categorized univariate and multivariate (logistic regression) analyses were performed to identify stone-free predictors at first FURSL. Results: Stone-free rate at first FURSL was $73.62 \%$. Predictors in males were patient age and stone size, density and multiplicity; in females, body mass index and multiplicity of stones. Conclusions: Stone-free rate predictors at first FURSL are different in males and females. Women with overweight and obesity probably have easy-to-fragment and easy-to-extract stones associated with uric acid.
\end{abstract}

KEY WORDS: Ureteroscopy. Kidney stones. Obesity. Costs and cost analysis.

\section{Introducción}

La ureteroscopia flexible con litotricia láser (URSLL) es el tratamiento mínimamente invasivo usado con mayor frecuencia en pacientes con cálculos renales que tienen factores de mal pronóstico para la litotricia extracorpórea o en quienes falló este tratamiento. Además, es útil en pacientes con discrasias sanguíneas o comorbilidades que impiden la realización de una nefrolitotricia percutánea para cálculos voluminosos.

El objetivo primario en todo procedimiento quirúrgico para cálculos renales debería ser la eliminación
Fecha de recepción: 15-08-2018

Fecha de aceptación: 05-01-2019

DOI:10.24875/GMM.19004569
Gac Med Mex. 2019;155:162-167

Disponible en PubMed www.gacetamedicademexico.com 
completa de la carga litiásica en el primer intento; el grado en el que el procedimiento logre este objetivo es un criterio importante para guiar la decisión y expectativas del paciente. ${ }^{1}$

Al ser un procedimiento sin heridas y técnicamente menos demandante que la nefrolitotricia percutánea, la URSLL es solicitada por el paciente directamente o sugerida por el médico en un intento de generalización de tratamiento quirúrgico para todos los tipos de cálculos, lo que genera que no todos los cálculos renales se resuelvan en la primera cirugía y que incluso en algunos casos sean necesarios tres o cuatro procedimientos de este tipo para resolverlos, lo que se traduce en costos quirúrgicos elevados, innecesarios y evitables si se hubiera optado por un procedimiento quirúrgico distinto desde el inicio.

En un intento por seleccionar adecuadamente a los pacientes para URSLL, varios estudios han buscado los factores que predicen el estado libre de cálculos (ELC), pero sin tomar en consideración el número de procedimientos necesarios para su resolución. ${ }^{2}$

Entre los factores más frecuentemente identificados como predictores de ELC en la URSLL están la localización, la multiplicidad y el volumen del cálculo. ${ }^{2}$

No existen estudios que evalúen específicamente estos factores en cada sexo, por lo que los resultados son una mezcla de factores pronósticos asumiendo que son los mismos para hombres y mujeres, sin tomar en consideración que existen características distintas entre ambos, como la composición y, por ende, la dureza del cálculo, el tamaño del riñón y las características estructurales distintivas de los genitales en uno y otro sexo.

Por lo anterior, conocer los factores predictores de ELC en un solo tiempo quirúrgico en pacientes sometidos a URSLL ayudará a tomar mejores decisiones terapéuticas basadas en costo-efectividad.

\section{Método}

Se estudió una cohorte retrospectiva de pacientes consecutivos sometidos a URSLL en el Hospital de Especialidades del Centro Médico Nacional Siglo XXI, Instituto Mexicano del Seguro Social, de octubre de 2014 a enero de 2017, que incluyó a hombres y mujeres mayores de 18 años con cálculos renales que tuvieran tomografía computarizada abdominopélvica y placa simple de abdomen preoperatoria y estudios radiológicos de control para evaluar la resolución de los cálculos. Se excluyeron pacientes sometidos a URSLL previamente para el mismo cálculo, aquellos con cálculos no observables en la placa simple de abdomen preoperatoria y quienes tuvieran datos incompletos en el seguimiento. En todos se utilizó fluoroscopia transoperatoria, láser de Holmio para la fragmentación (fibra de 200 micras) y se colocó un catéter $\mathrm{JJ}$ al final del procedimiento a discreción del cirujano, que permaneció por lo menos hasta el estudio radiológico de control.

Las variables evaluadas como potenciales predictores fueron edad, sexo, índice de masa corporal, antecedente de infección urinaria, antecedente de cirugías urológicas endoscópicas o abiertas por cálculos en el mismo riñón, antecedente de estrecheces urinarias o malformaciones obstructivas de tracto urinario superior, tamaño del cálculo (suma del diámetro mayor de todos los cálculos), densidad (valor más alto de unidades Hounsfield), localización de los cálculos, multiplicidad, longitud y diámetro infundibular del cáliz inferior y ángulo pielocalicial.

El desenlace primario evaluado fue el ELC, definido como litos ausentes o no significativos $(<4 \mathrm{~mm}$ ) en el estudio de imagen de control (nivel $4 \mathrm{C}$ y $4 \mathrm{x}$ de Somani), tres semanas después del primer procedimiento de URSLL. ${ }^{3}$

EI análisis estadístico se realizó con SPSS versión 20. Los pacientes se dividieron por sexo. En el análisis univariado se contrastó éxito versus fracaso para ELC mediante t de Student o $U$ de Mann-Whitney de acuerdo con la distribución de los datos. Para las variables categóricas o estratificadas, el contraste de hipótesis se realizó con chi cuadrada.

Se realizó un análisis multivariado mediante regresión logística con el método de Wald, con las variables predictivas encontradas en el análisis univariado para identificar interacciones y ajustar cada una. Se obtuvieron las razones de momios (RM) y los intervalos de confianza de las variables que se mantuvieron como predictores.

No existieron maniobras, procedimientos o condiciones de riesgo adicionales al tratamiento asignado previamente al paciente, por lo que se consideró como una investigación de riesgo menor al mínimo, la cual fue aprobada por el Comité Local de Ética e Investigación del Hospital con registro R-2016-3601-93.

\section{Resultados}

Se obtuvieron 158 pacientes evaluables (Figura 1), de los cuales 93 (58.9\%) fueron mujeres. La edad de la población fue de 50.5 años en promedio. La media de peso fue de $73.7 \mathrm{~kg}$, talla de $1.60 \mathrm{~m}$ e índice de masa corporal de 28.5; 114 pacientes tuvieron sobrepeso u obesidad, 85 (53.2 \%) eran cálculos del lado 


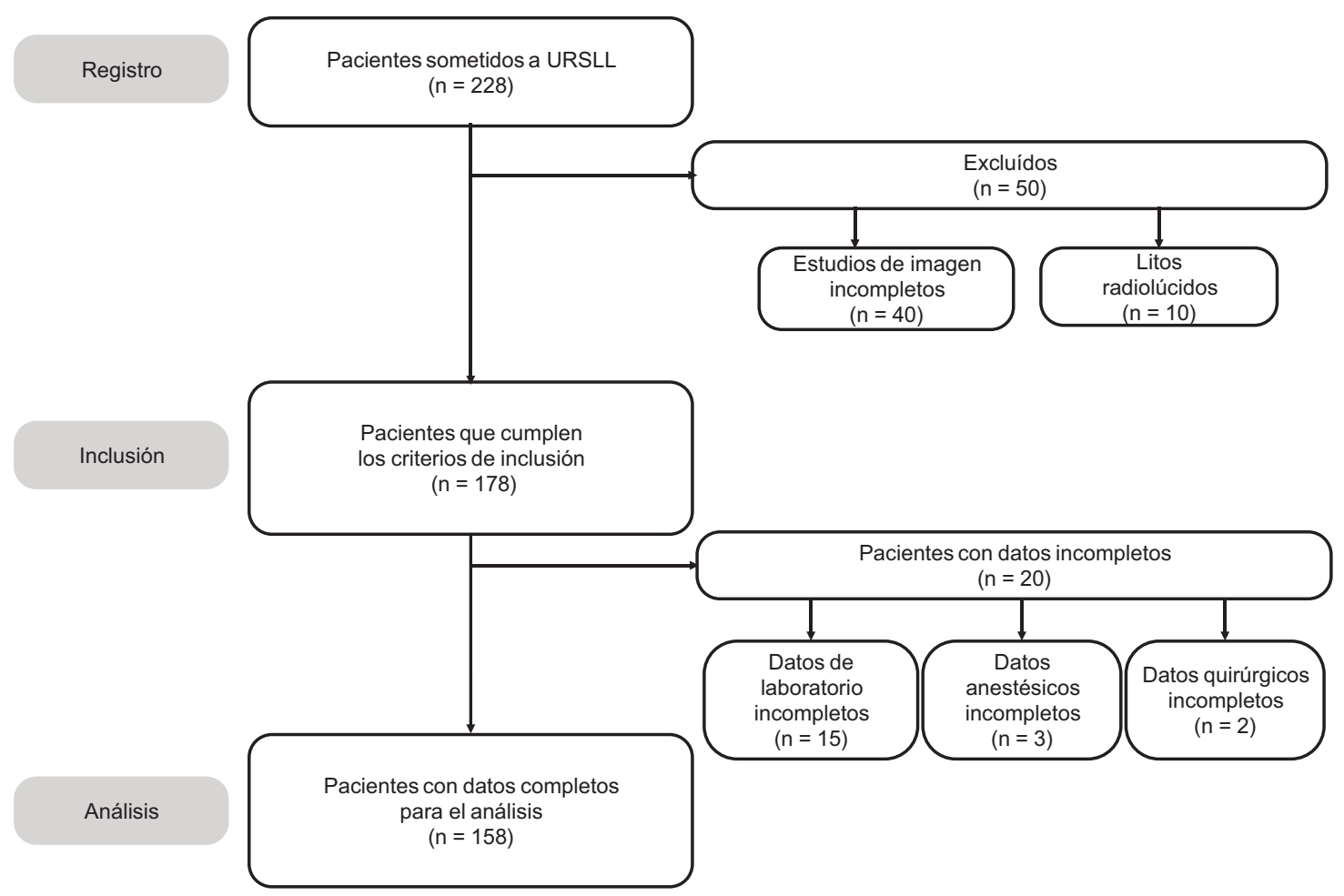

Figura 1. Diagrama de flujo de la cohorte de pacientes sometidos a URSLL.

izquierdo y 98 (62.02\%) eran únicos. Los cálculos se localizaron en cáliz superior en nueve (5.7\%), cáliz medio en siete $(4.4 \%)$, cáliz inferior en $64(40.5 \%)$, pelvis renal en 14 (8.9\%), uréter en 10 (6.3\%), múltiples litos que no incluían el cáliz inferior ocho (5.1\%) y múltiples litos que incluían el cáliz inferior $46(29.1 \%)$. La media del tamaño de los cálculos fue de $16.04 \mathrm{~mm}$ (Tabla 1). Se utilizó camisa ureteral en $132(83.5 \%)$ y se dejó un catéter JJ en 149 (94.3\%). EI ELC en general fue de $73.62 \%$.

En el análisis global univariado (ambos sexos) para ELC se encontraron como predictores el número (único versus múltiple) y el volumen de los cálculos. En el análisis categorizado por sexo, los factores pronósticos fueron distintos para hombres y mujeres. En el análisis multivariado, en los hombres se mantuvieron como predictores para litiasis residual posterior al primer procedimiento la edad, localización, tamaño y densidad de los cálculos; en las mujeres, el índice de masa corporal normal o bajo y la litiasis múltiple predijeron la litiasis residual (Tabla 2).

\section{Discusión}

En el Hospital de Especialidades del Centro Médico Nacional Siglo XXI, la URSLL no es habitual para los cálculos del tercio superior del uréter; la mayoría son tratados sin la necesidad de instrumentos flexibles, por lo que nuestros resultados podrían aplicarse mejor a pacientes que solo tienen cálculos renales. El estudio multicéntrico de la Clinical Research Office of the Endourological Society registró $73 \%$ de pacientes con cálculos que solo involucraban el uréter y registró juntos los resultados de procedimientos de ureteroscopia rígida y flexible, ${ }^{4}$ lo que hace difícil obtener conclusiones generalizables al riñón.

Resorlu et al. describieron que el tamaño, composición y multiplicidad de los cálculos, así como el ángulo infundibulopiélico y la presencia de malformaciones renales eran factores pronósticos para ELC en la URSLL, ${ }^{2}$ a los que lto et al. agregaron la hidronefrosis. ${ }^{5}$ En el análisis global de los pacientes solo el volumen y la multiplicidad tuvieron influencia en el ELC, sin embargo, en un análisis multivariado separando a los pacientes por sexo encontramos que los factores que predicen el ELC en la primera URSLL son distintos en hombres y mujeres. Son de pronóstico favorable para los hombres, la edad menor a 50 años, la localización en el uréter o en los polos renales, y una menor densidad y tamaño del cálculo. Sorprendentemente, en las mujeres solo el índice de masa corporal $\geq 25$ y los cálculos únicos influyeron positivamente.

Es probable que la mayor proporción de hombres en las cohortes de otros estudios ${ }^{4}$ influya en los resultados 
Tabla 1. Características de los pacientes sometidos a ureteroscopia flexible con litotricia láser $(n=158)$

\begin{tabular}{|c|c|c|}
\hline Característica & $\mathbf{n}$ & $\%$ \\
\hline \multicolumn{3}{|l|}{ Demográficas } \\
\hline Mujeres & 93 & 58.9 \\
\hline Obesidad o sobrepeso & 114 & 72.2 \\
\hline Lado izquierdo & 85 & 53.8 \\
\hline Cálculo único & 98 & 62 \\
\hline \multicolumn{3}{|l|}{ Localización del cálculo } \\
\hline Cáliz superior & 9 & 5.7 \\
\hline Cáliz medio & 7 & 4.4 \\
\hline Cáliz inferior & 64 & 40.5 \\
\hline Piélico & 14 & 8.9 \\
\hline Ureteral & 10 & 6.3 \\
\hline Múltiple que no incluye el cáliz inferior & 8 & 5.1 \\
\hline Múltiple que incluye el cáliz inferior & 46 & 29.1 \\
\hline \multicolumn{3}{|l|}{ Clínicas preoperatorias } \\
\hline Antecedente de infección de vías urinarias & 29 & 18.4 \\
\hline Antecedente de cirugía abierta & 33 & 20.9 \\
\hline Antecedente de cirugía endoscópica & 78 & 49.4 \\
\hline Estrechez ureteral o malformaciones & 29 & 18.4 \\
\hline Uso de aspirina o anticoagulantes & 7 & 4.4 \\
\hline Cirrosis hepática & 3 & 1.9 \\
\hline Hipertensión arterial & 38 & 24.1 \\
\hline Hematuria preoperatoria & 114 & 72.2 \\
\hline \multicolumn{3}{|l|}{ Urocultivo } \\
\hline Negativo & 109 & 69 \\
\hline Escherichia coli no BLEE & 16 & 10.1 \\
\hline Escherichia coli BLEE & 26 & 16.5 \\
\hline Enterococcus sp. & 2 & 1.3 \\
\hline Klebsiella sp. & 1 & 0.6 \\
\hline Pseudomonas sp. & 4 & 2.5 \\
\hline Portador de catéter JJ & 65 & 41.1 \\
\hline \multicolumn{3}{|l|}{ Transoperatorias } \\
\hline Uso de camisa ureteral & 132 & 83.5 \\
\hline \multicolumn{3}{|l|}{ Tipo de anestesia } \\
\hline Bloqueo & 7 & 4.4 \\
\hline Bloqueo más general & 5 & 3.2 \\
\hline General & 146 & 92.4 \\
\hline Hipertensión transoperatoria & 10 & 6.3 \\
\hline \multirow[t]{2}{*}{ Colocación de catéter JJ al final } & 149 & 94.3 \\
\hline & Media & DE \\
\hline Edad (años) & 50.52 & 11.68 \\
\hline Índice de masa & 28.55 & 5.34 \\
\hline Creatinina & 0.98 & 0.42 \\
\hline Tamaño del cálculo (mm) & 16.04 & 10.15 \\
\hline
\end{tabular}

(Continúa)
Tabla 1. Características de los pacientes sometidos a ureteroscopia flexible con litotricia láser $(n=158)$ (Continuación)

\begin{tabular}{lcc}
\hline Característica & Media & $\%$ \\
\hline Densidad del cálculo (UH) & 1106.03 & 347.78 \\
Ángulo pielocalicial (grados) & 82.78 & 14.92 \\
Diámetro infundíbulo inferior $(\mathrm{mm})$ & 10.51 & 9.80 \\
Longitud infundíbulo inferior $(\mathrm{mm})$ & 19.25 & 6.17 \\
\hline BLEE = betalactamasa de espectro extendido, UH = unidades Hounsfield. & \\
Nota: El tamaño del cálculo es la suma de los diámetros mayores de todos los cálculos.
\end{tabular}

de los análisis globales (no categorizados por sexo), haciendo que los factores pronósticos de mayor peso sean los que tienen importancia en los hombres y ocultando los significativos en las mujeres.

La URSLL es una opción terapéutica adecuada en pacientes con obesidad, ${ }^{6}$ pero la influencia del índice de masa corporal sobre el ELC es contradictoria. ${ }^{7-10}$ Doizi et al. encontraron que un mayor índice de masa corporal se asociaba con mayor resolución de los cálculos en el primer procedimiento, incluso más evidente al realizar la comparación con pacientes con alto índice de masa corporal. ${ }^{8}$ En forma similar, en nuestro estudio las mujeres con índice de masa corporal $\geq 25$ tuvieron un pronóstico favorable para ELC.

La frecuencia de litiasis urinaria aumenta con el índice de masa corporal, independientemente de la edad, lo que es más evidente en las mujeres. ${ }^{11} \mathrm{~A}$ medida que aumenta el índice de masa corporal aumenta también la proporción de cálculos de ácido úrico en ambos sexos, ${ }^{12}$ lo que ha sido más evidente en las últimas dos décadas en las mujeres. ${ }^{13}$ Creemos que los cálculos en las mujeres con obesidad y sobrepeso son más blandos, lo que se traduciría en un mejor pronóstico con la resolución endoscópica por la mayor velocidad de fragmentación-extracción de estos cuando se comparan con cálculos de contenido cálcico. ${ }^{12,14-16}$

Daudon et al. registraron que la frecuencia de cálculos de ácido úrico en mujeres con peso normal aumentaba de 6.1 a $17.1 \%$ en mujeres con obesidad, además de disminuir la proporción de pacientes con cálculos de fosfato de calcio. ${ }^{17}$ También Siener et al. encontraron que a medida que aumentaba el índice de masa corporal de las mujeres, disminuía la proporción de cálculos de oxalato de calcio y aumentaba la excreción urinaria de uratos. ${ }^{18}$

En relación con el punto de corte para considerar el ELC, Ghani et al. consideran que el límite debe ser $2 \mathrm{~mm}$, sin embargo, también se hace referencia a que el estudio para discriminar un umbral tan fino tendría que ser una tomografía computarizada debido a la 
Tabla 2. Predictores de litiasis residual en la primera ureteroscopia flexible con litotricia láser categorizados por sexo $(n=158)$

\begin{tabular}{|c|c|c|c|c|c|c|c|c|c|c|c|c|c|c|}
\hline \multirow[t]{4}{*}{ Variables } & \multicolumn{7}{|c|}{ Hombres $(n=65)$} & \multicolumn{7}{|c|}{ Mujeres $(n=93)$} \\
\hline & \multicolumn{5}{|c|}{ Univariado* } & \multicolumn{2}{|c|}{ Multivariado ${ }^{\star *}$} & \multicolumn{5}{|c|}{ Univariado* } & \multicolumn{2}{|c|}{ Multivariado**} \\
\hline & \multicolumn{2}{|c|}{ Resuelta } & \multicolumn{2}{|c|}{ Persistente } & \multirow[t]{2}{*}{ p } & \multirow[t]{2}{*}{ RM ajustada } & \multirow[t]{2}{*}{$\mathrm{p}$} & \multicolumn{2}{|c|}{ Resuelta } & \multicolumn{2}{|c|}{ Persistente } & \multirow[t]{2}{*}{$p$} & \multirow{2}{*}{$\begin{array}{c}\text { RM } \\
\text { ajustada }\end{array}$} & \multirow[t]{2}{*}{$p$} \\
\hline & $\mathrm{n}$ & $\%$ & $\mathrm{n}$ & $\%$ & & & & $\mathrm{n}$ & $\%$ & $\mathbf{n}$ & $\%$ & & & \\
\hline \multicolumn{15}{|l|}{ Edad (años) } \\
\hline$\leq 50$ & 17 & 44.7 & 4 & 14.8 & 0.015 & 1 & 0.009 & 28 & 52.8 & 19 & 47.5 & 0.611 & & \\
\hline$>50$ & 21 & 55.3 & 23 & 85.2 & & $\begin{array}{c}39.80 \\
(2.52-627.16)\end{array}$ & & 25 & 47.2 & 21 & 52.5 & & & \\
\hline \multicolumn{15}{|l|}{ Índice de masa corporal } \\
\hline$<25$ & 10 & 26.3 & 6 & 22.2 & 0.706 & & & 10 & 18.9 & 18 & 45.0 & 0.007 & $\begin{array}{c}4.72 \\
(1.63-13.66)\end{array}$ & 0.004 \\
\hline$\geq 25$ & 28 & 73.7 & 21 & 77.8 & & & & 43 & 81.1 & 22 & 55.0 & & 1 & \\
\hline \multicolumn{15}{|l|}{ Multiplicidad } \\
\hline Único & 21 & 55.3 & 16 & 59.3 & 0.749 & & & 43 & 81.1 & 18 & 45.0 & 0.000 & 1 & 0.000 \\
\hline Múltiple & 17 & 44.7 & 11 & 40.7 & & & & 10 & 18.9 & 22 & 55.0 & & $\begin{array}{c}9.06 \\
(1.95-27.83)\end{array}$ & \\
\hline \multicolumn{15}{|c|}{ Localización (litos únicos) } \\
\hline Ureteral & 6 & 15.8 & 1 & 3.7 & 0.115 & 1 & 0.018 & 2 & 3.8 & 1 & 2.5 & 0.002 & & \\
\hline Calicial (polar) & 15 & 39.5 & 11 & 40.7 & & 71.32 & 0.004 & 32 & 60.4 & 15 & 37.5 & & & \\
\hline $\begin{array}{l}\text { Calicial interpolar o } \\
\text { piélico }\end{array}$ & 2 & 5.3 & 6 & 22.2 & & $\begin{array}{c}(2.09-240.72) \\
1525.72 \\
(10.10-230297.25)\end{array}$ & & & & 3 & 1.0 & & & \\
\hline \multicolumn{15}{|l|}{ Tamaño cálculo (mm) } \\
\hline$\leq 10$ & 17 & 44.7 & 5 & 18.5 & 0.088 & 1 & 0.03 & 21 & 39.6 & 19 & 25.0 & 0.04 & & \\
\hline $11-20$ & 11 & 28.9 & 11 & 40.7 & & 26.70 & 0.01 & 24 & 45.3 & 15 & 37.5 & & & \\
\hline$>20$ & 10 & 26.3 & 11 & 40.7 & & $\begin{array}{c}(1.37-518.36) \\
38.35 \\
(2.35-624.07)\end{array}$ & & 8 & 15.1 & 15 & 37.5 & & & \\
\hline \multicolumn{15}{|c|}{$\begin{array}{l}\text { Antecedente de infección } \\
\text { de vías urinarias }\end{array}$} \\
\hline No & 37 & 97.4 & 26 & 96.3 & 1.00 & & & 41 & 77.4 & 25 & 62.5 & 0.118 & & \\
\hline Sí & 1 & 2.6 & 1 & 3.7 & & & & 12 & 22.6 & 15 & 37.5 & & & \\
\hline $\begin{array}{l}\text { Antecedente de cirug } \\
\text { abierta }\end{array}$ & & & & & & & & & & & & & & \\
\hline No & 31 & 81.6 & 19 & 70.4 & 0.291 & & & 41 & 77.4 & 34 & 85.0 & 0.356 & & \\
\hline Sí & 7 & 18.4 & 8 & 29.6 & & & & 12 & 22.6 & 6 & 15.0 & & & \\
\hline $\begin{array}{l}\text { Antecedente de cirug } \\
\text { endoscópica }\end{array}$ & & & & & & & & & & & & & & \\
\hline No & 20 & 52.6 & 21 & 77.8 & 0.038 & & & 22 & 41.5 & 17 & 42.5 & 0.924 & & \\
\hline Sí & 18 & 47.4 & 6 & 22.2 & & & & 31 & 58.5 & 23 & 57.5 & & & \\
\hline $\begin{array}{l}\text { Ángulo } \\
\text { pielocalicial (grados) }\end{array}$ & & & & & & & & & & & & & & \\
\hline$\leq 100$ & 36 & 94.7 & 23 & 85.2 & 0.224 & & & 49 & 92.5 & 38 & 95.0 & 0.696 & & \\
\hline$>100$ & 2 & 5.3 & 4 & 14.8 & & & & 4 & 7.5 & 2 & 5.0 & & & \\
\hline Unidades Hounsfield & & & & & & & & & & & & & & \\
\hline$\leq 850$ & 13 & 34.2 & 3 & 11.1 & & 1 & 0.044 & 12 & 22.6 & 8 & 20.0 & 0.488 & & \\
\hline $851-1450$ & 23 & 60.5 & 17 & 63.0 & & $\begin{array}{c}12.07 \\
(1.06-136.57)\end{array}$ & 0.039 & 33 & 62.3 & 22 & 55.0 & & & \\
\hline$>1450$ & 2 & 5.3 & 7 & 25.9 & & $\begin{array}{c}22.66 \\
(1.16-442.20)\end{array}$ & & 8 & 15.1 & 10 & 25.0 & & & \\
\hline
\end{tabular}


disminución de la sensibilidad de la placa simple de abdomen a medida que los cálculos son más pequeños. ${ }^{1}$

Someter a todos los pacientes a tomografía computarizada de control no es habitual en los estudios internacionales, en los que existe una mezcla válida, por lo menos en la práctica, de ultrasonido, placas de abdomen y tomografía computarizada, estudios heterogéneos a partir de los cuales Ghani obtiene sus conclusiones y emite sus recomendaciones. ${ }^{1}$

A pesar de su sensibilidad, el uso de la tomografía computarizada se ha visto limitado por la necesidad de mantener la exposición a la radiación tan baja como sea posible (concepto ALARA) y la probabilidad de que en el futuro existan otros eventos de litiasis en estos pacientes o varias cirugías para resolver el mismo cálculo. ${ }^{19}$

La discriminación de fragmentos $<4 \mathrm{~mm}$ en una placa simple de abdomen es complicada, por lo que disminuir el punto de corte a $2 \mathrm{~mm}$ o menos solo agregaría subjetividad y no modificaría el tratamiento a seguir para los cálculos residuales, que esencialmente es el mismo si el cálculo mide 2 o $3 \mathrm{~mm}$, como lo muestran las guías internacionales, en las que se indica que $>5 \mathrm{~mm}$ es el punto de corte a partir del cual aumenta la probabilidad para requerir un tratamiento invasivo adicional para los cálculos residuales no infectados. Lo anterior se basa en la historia natural de estos fragmentos y en su probabilidad de expulsión espontánea. ${ }^{20,21}$

Pretendemos que nuestro estudio con el punto de corte a $4 \mathrm{~mm}$ tenga validez externa, es decir, que pueda generalizarse a la práctica urológica habitual para la toma de decisiones conforme a las guías internacionales vigentes hasta este momento, manteniendo un balance costo-efectividad-seguridad.

Nuestra investigación es la primera que demuestra que los factores pronósticos de ELC son distintos en hombres y mujeres, por lo que consideramos que es un error generalizar los factores pronósticos en los pacientes con litiasis sin tomar en consideración el sexo. Sugerimos que nuestros hallazgos se validen con otros estudios clínicos para poder generalizarlos.

Las nuevas tecnologías deberían evaluarse en el contexto de costo-efectividad y no conforme preferencias personales. El objetivo en todo procedimiento quirúrgico debería ser la resolución del problema en el primer intento, por lo que debería estandarizarse como el desenlace a evaluar en futuros estudios.

\section{Bibliografía}

1. Ghani KR, Wolf JS. What is the stone-free rate following flexible ureteroscopy for kidney stones? Nat Rev Urol. 2015;12:281-288.

2. Resorlu B, Unsal A, Gulec H, Oztuna D. A new scoring system for predicting stone-free rate after retrograde intrarenal surgery: the "resorlu-unsal stone score". Urology. 2012;80:512-518.

3. Somani BK, Desai M, Traxer O, Lahme S. Stone-free rate (SFR): a new proposal for defining levels of SFR. Urolithiasis. 2014;42:95.

4. De la Rosette J, Denstedt J, Geavlete P, Keeley F, Matsuda T, Pearle M, et al. The clinical research office of the endourological society ureteroscopy global study: indications, complications, and outcomes in 11,885 patients. J Endourol. 2014;28:131-139.

5. Ito H, Sakamaki K, Kawahara T, Terao H, Yasuda K, Kuroda S, et al. Development and internal validation of a nomogram for predicting stone-free status after flexible ureteroscopy for renal stones. BJU Int. 2015;115:446-451.

6. Ishii H, Couzins M, Aboumarzouk O, Biyani CS, Somani BK. Outcomes of systematic review of ureteroscopy for stone disease in the obese and morbidly obese population. J Endourol. 2016;30:135-145.

7. Schott F, Knipper S, Orywal AK, Gross AJ, Netsch C. Effect of the body mass index on outcomes of ureterorenoscopy for renal stones. Urologe A. 2016;55:1462-1469.

8. Doizi S, Letendre J, Bonneau C, Gil Diez-De Medina S, Traxer O. Comparative study of the treatment of renal stones with flexible ureterorenoscopy in normal weight, obese, and morbidly obese patients. Urology. 2015;85:38-44.

9. Dash A, Schuster TG, Hollenbeck BK, Faerber GJ, Wolf JS. Ureteroscopic treatment of renal calculi in morbidly obese patients: a stone-matched comparison. Urology. 2002;60:393-397.

10. Laclergerie F, Jacquemet B, Guichard G, Bernardini S, Chabannes E, Martin L, et al. Flexible ureterorenoscopy in obese patients: results from a large monocenter cohort. Prog Urol. 2014;24:634-639.

11. Curhan GC, Willett WC, Rimm EB, Speizer FE, Stampfer MJ. Body size and risk of kidney stones. J Am Soc Nephrol. 1998;9:1645-1652.

12. Trinchieri A, E. Croppi E, Montanari E. Obesity and urolithiasis: evidence of regional influences. Urolithiasis. 2017:45:271-278.

13. Moses R, Pais VM, Ursiny M, Prien EL, Miller N, Eisner BH. Changes in stone composition over two decades: evaluation of over 10,000 stone analyses. Urolithiasis. 2015;43:135-139.

14. Xu LHR, Adams-Huet B, Poindexter JR, Maalouf NM, Moe OW, Sakhaee K. Temporal changes in kidney stone composition and in risk factors predisposing to stone formation. J Urol. 2017;197:1465-1471.

15. Spivacow FR, Del Valle EE, Lores E, Rey PG. Kidney stones: composition, frequency and relation to metabolic diagnosis. Medicina (B Aires). 2016;76:343-348.

16. Sorokin I, Cardona-Grau DK, Rehfuss A, Birney A, Stavrakis C, Leinwand $G$, et al. Stone volume is best predictor of operative time required in retrograde intrarenal surgery for renal calculi: implications for surgical planning and quality improvement. Urolithiasis. 2016;44:545-550.

17. Daudon $M$, Lacour $B$, Jungers $P$. Influence of body size on urinary stone composition in men and women. Urol Res. 2006;34:193-199.

18. Siener R, Glatz S, Nicolay C, Hesse A. The role of overweight and obesity in calcium oxalate stone formation. Obes Res. 2004;12:106-113.

19. Lukasiewicz A, Bhargavan-Chatfield M, Coombs L, Ghita M, Weinreb J, Gunabushanam G, et al. Radiation dose index of renal colic protocol CT studies in the United States: a report from the American College of Radiology National Radiology Data Registry. Radiology. 2014;271:445-451.

20. Rebuck DA, Macejko A, Bhalani V, Ramos P, Nadler RB. The natural history of renal stone fragments following ureteroscopy. Urology. 2011;77:564-568.

21. Candau C, Saussine C, Lang H, Roy C, Faure F, Jacqmin D. Natural history of residual renal stone fragments after ESWL. Eur Urol. 2000;37:18-22. 